\title{
Integrated genomic analyses of ovarian carcinoma
}

\author{
The Cancer Genome Atlas Research Network*
}

A catalogue of molecular aberrations that cause ovarian cancer is critical for developing and deploying therapies that will improve patients' lives. The Cancer Genome Atlas project has analysed messenger RNA expression, microRNA expression, promoter methylation and DNA copy number in 489 high-grade serous ovarian adenocarcinomas and the DNA sequences of exons from coding genes in 316 of these tumours. Here we report that high-grade serous ovarian cancer is characterized by TP53 mutations in almost all tumours $(96 \%)$; low prevalence but statistically recurrent somatic mutations in nine further genes including NF1, BRCA1, BRCA2, RB1 and CDK12; 113 significant focal DNA copy number aberrations; and promoter methylation events involving 168 genes. Analyses delineated four ovarian cancer transcriptional subtypes, three microRNA subtypes, four promoter methylation subtypes and a transcriptional signature associated with survival duration, and shed new light on the impact that tumours with BRCA1/2 (BRCA1 or $B R C A 2)$ and CCNE1 aberrations have on survival. Pathway analyses suggested that homologous recombination is defective in about half of the tumours analysed, and that NOTCH and FOXM1 signalling are involved in serous ovarian cancer pathophysiology.

Ovarian cancer is the fifth-leading cause of cancer death among women in the United States; 21,880 new cases and 13,850 deaths were estimated to have occurred in $2010^{1}$. Most deaths $(\sim 70 \%)$ are of patients presenting with advanced-stage, high-grade serous ovarian cancer $^{2,3}$ (HGS-OvCa). The standard treatment is aggressive surgery followed by platinum-taxane chemotherapy. After therapy, platinumresistant cancer recurs in approximately $25 \%$ of patients within six months ${ }^{4}$, and the overall five-year survival probability is $31 \%$ (ref. 5). Approximately $13 \%$ of $\mathrm{HGS}-\mathrm{OvCa}$ is attributable to germline mutations in BRCA1/2 (refs 6,7), and a smaller percentage can be accounted for by other germline mutations. However, most ovarian cancer can be attributed to a growing number of somatic aberrations ${ }^{8}$.

The lack of successful treatment strategies led the Cancer Genome Atlas (TCGA) researchers to measure comprehensively genomic and epigenomic abnormalities on clinically annotated HGS-OvCa samples to identify molecular abnormalities that influence pathophysiology, affect outcome and constitute therapeutic targets. Microarray analyses produced high-resolution measurements of mRNA expression, microRNA (miRNA) expression, DNA copy number and DNA promoter methylation for 489 HGS-OvCa tumours, and massively parallel sequencing coupled with hybrid affinity capture ${ }^{9,10}$ provided wholeexome DNA sequence information for 316 of these samples.

\section{Samples and clinical data}

This Article reports the analysis of 489 clinically annotated stage-IIIV HGS-OvCa samples and corresponding normal DNA (Supplementary Methods, section 1, and Supplementary Table 1.1). Patients reflected the age at diagnosis, stage, tumour grade and surgical outcome of individuals typically diagnosed with HGS-OvCa. Clinical data were current as of 25 August 2010. HGS-OvCa specimens were surgically resected before systemic treatment but all patients received a platinum agent and $94 \%$ received a taxane. The median progression-free survival and overall survival of the cohort are similar to those in previously published trials ${ }^{11,12}$. Twenty-five per cent of the patients remained free from disease and $45 \%$ were alive at the time of last follow-up, whereas $31 \%$ experienced disease progression within six months of completing platinum-based therapy. The median follow-up time was 30 months (range, 0-179 months). Samples for TCGA analysis were selected to have $>70 \%$ tumour cell nuclei and $<20 \%$ necrosis.

Coordinated molecular analyses using multiple molecular assays at independent sites were carried out as listed in Table 1 . The data set analysed here is available at the TCGA website (http://tcga-data.nci. nih.gov/docs/publications/ov_2011), in two tiers: open access and controlled access. Open-access data sets are publicly available, whereas controlled-access data sets, which include clinical or genomic information that could identify an individual, require user certification as described on the aforementioned website.

Table 1 | Characterization platforms used and data produced

\begin{tabular}{|c|c|c|c|}
\hline Data type & Platforms & Cases & Data access \\
\hline \multirow[t]{2}{*}{ DNA sequence of exome } & Illumina GAllx*† & 236 & Controlled \\
\hline & ABI SOLID* & 80 & Controlled \\
\hline Mutations present in exome & & 316 & Open \\
\hline \multirow[t]{5}{*}{ DNA copy number/genotype } & Agilent 244K§ \| & 97 & Open \\
\hline & Agilent 415K§ & 304 & Open \\
\hline & Agilent $1 \mathrm{M} \|$ & 539 & Open \\
\hline & Illumina 1MDUO & 535 & Controlled \\
\hline & Affymetrix SNP6* & 514 & Controlled \\
\hline \multirow[t]{3}{*}{ mRNA expression profiling } & Affymetrix U133A* & 516 & Open \\
\hline & Affymetrix Exon\# & 517 & Controlled \\
\hline & Agilent $244 K^{* *}$ & 540 & Open \\
\hline Integrated mRNA expression & & 489 & Open \\
\hline miRNA expression profiling & Agilent** & 541 & Open \\
\hline CpG DNA methylation & Illumina $27 \mathrm{~K}+\dagger$ & 519 & Open \\
\hline Integrative analysis & & 489 & Open \\
\hline Integrative analysis with & & 309 & Open \\
\hline
\end{tabular}

mutations

Production centres: *Broad Institute, †Washington University School of Medicine, *Baylor College of Medicine, §Harvard Medical School, ॥Memorial Sloan-Kettering Cancer Center, $₫$ HudsonAlpha

Institute for Biotechnology, \#Lawrence Berkeley National Laboratory, **University of North Carolina at Chapel Hill, ††University of Southern California. Extra data are available for many of these data types at the TCGA data coordinating centre. 


\section{Mutation analysis}

We performed exome capture and sequencing on DNA isolated from 316 HGS-OvCa samples and from matched normal samples for each individual (Supplementary Methods, section 2). Capture reagents targeted $\sim 180,000$ exons from $\sim 18,500$ genes totalling $\sim 33$ megabases of non-redundant sequence. Massively parallel sequencing on the Illumina GAIIx platform (236 sample pairs) or ABI SOLiD 3 platform (80 sample pairs) yielded $\sim 14$ gigabases per sample $\left(\sim 9 \times 10^{12}\right.$ bases in total). On average, $76 \%$ of coding bases were covered in sufficient depth in both the tumour and the matched normal samples to allow confident mutation detection (Supplementary Methods, section 2, and Supplementary Fig. 2.1). We annotated 19,356 somatic mutations ( $~ 61$ per tumour); these are classified in Supplementary Table 2.1. Mutations that may be important in HGSOvCa pathophysiology were identified by searching for nonsynonymous or splice site mutations present at significantly increased frequencies relative to background, by comparing mutations in this study to those in the Catalogue of Somatic Mutations in Cancer and Online Mendelian Inheritance in Man, and by predicting the mutations' impacts on protein function.

Two different algorithms (Supplementary Methods, section 2) identified nine genes (Table 2) for which the number of nonsynonymous or splice site mutations was significantly more than that expected on the basis of mutation distribution models. Consistent with published results ${ }^{13}$, TP53 was mutated in 303 of 316 samples ( 283 by automated methods and 20 after manual review), and BRCA1 and BRCA2 had germline mutations in $9 \%$ and $8 \%$ of cases, respectively, and showed somatic mutations in a further $3 \%$ of cases. We identified six other statistically recurrently mutated genes: $R B 1$, NF1, FAT3, CSMD3, GABRA6 and CDK12. CDK12 is involved in RNA splicing regulation ${ }^{14}$ and was previously implicated in lung and large-intestine tumours ${ }^{15,16}$. Five of the nine CDK12 mutations were either nonsense or indel, suggesting potential loss of function, and the four missense mutations (Arg882Leu, Tyr901Cys, Lys975Glu and Leu996Phe) were clustered in its protein kinase domain. GABRA6 and FAT3 both appeared as significantly mutated but did not seem to be expressed in HGS-OvCa (Supplementary Fig. 2.1) or fallopian tube tissue, so it is less likely that mutation of these genes has a significant role in HGS-OvCa.

We compared mutations from this study with mutations in the Catalogue of Somatic Mutations in Cancer $^{17}$ and Online Mendelian Inheritance in $\mathrm{Man}^{18}$ databases to identify more HGS-OvCa genes that are less commonly mutated. These comparisons yielded 477 and 211 matches, respectively (Supplementary Table 2.4), including mutations in BRAF (Asn581Ser), PIK3CA (Glu545Lys and His1047Arg), KRAS (Gly12Asp) and NRAS (Gln61Arg). These mutations have been shown to have transforming activity, so we believe that these mutations are rare but important drivers in HGS-OvCa.

We combined evolutionary information from sequence alignments of protein families and whole vertebrate genomes, predicted local protein structure and selected human SwissProt protein features

Table 2 | Significantly mutated genes in HGS-OvCa

\begin{tabular}{lccc}
\hline Gene & No. of mutations & No. validated & No. unvalidated \\
\hline TP53 & 302 & 294 & 8 \\
BRCA1 & 11 & 10 & 1 \\
CSMD3 & 19 & 19 & 0 \\
NF1 & 13 & 13 & 0 \\
CDK12 & 9 & 9 & 0 \\
FAT3 & 19 & 18 & 1 \\
GABRA6 & 6 & 6 & 0 \\
BRCA2 & 10 & 10 & 0 \\
RB1 & 6 & 6 & 0 \\
\hline
\end{tabular}

Validated mutations are those that have been confirmed with an independent assay. Most of them are validated using a second independent whole-genome-amplification sample from the same tumour.

Unvalidated mutations have not been independently confirmed but have a high likelihood to be true mutations. An extra 25 mutations in TP53 were observed by hand curation.
(Supplementary Methods, section 3) to identify putative driver mutations using $\mathrm{CHASM}^{19,20}$ after training on mutations in known oncogenes and tumour suppressors. CHASM identified 122 missense mutations predicted to be oncogenic (Supplementary Table 3.1). Mutation-driven changes in protein function were deduced from evolutionary information for all confirmed somatic missense mutations by comparing protein family sequence alignments and residue placement in known or homology-based three-dimensional protein structures using MutationAssessor (Supplementary Methods, section 4). Twenty-seven per cent of missense mutations were predicted to affect protein function (Supplementary Table 2.1).

\section{Copy number analysis}

Somatic copy number alterations (SCNAs) present in the 489 HGSOvCa genomes were identified and compared with glioblastoma multiforme data (Fig. 1a). SCNAs were divided into regional aberrations that affected extended chromosome regions and smaller focal aberrations (Supplementary Methods, section 5). A statistical analysis of regional aberrations ${ }^{21}$ (Supplementary Methods, section 5) identified eight recurrent gains and 22 losses, all of which have been reported previously $^{22}$ (Fig. 1b and Supplementary Table 5.1). Five of the gains and 18 of the losses occurred in more than $50 \%$ of the tumours.

We used GISTIC ${ }^{21,23}$ (Supplementary Methods, section 5) to identify recurrent focal SCNAs. This yielded 63 regions of focal amplification (Fig. 1c; Supplementary Methods, section 5; and Supplementary Table 5.2), including 26 that encoded eight or fewer genes. The most common focal amplifications encoded CCNE1, MYC and MECOM (Fig. 1c; Supplementary Methods, section 5; and Supplementary Table 5.2), each of which was highly amplified in more than $20 \%$ of tumours. New tightly localized amplification peaks in HGS-OvCa encoded the receptor for activated C-kinase, ZMYND8; the p53 target gene IRF2BP2; the DNA-binding protein inhibitor ID4; the embryonic development gene $P A X 8$; and the telomerase catalytic subunit, TERT. Three data sources-Ingenuity Systems (http://www. ingenuity.com/), ClinicalTrials.gov (http://clinicaltrials.gov) and DrugBank (http://www.drugbank.ca)-were used to identify possible therapeutic inhibitors of amplified, overexpressed genes. From this search, we found that 22 genes that are therapeutic targets, including $M E C O M, M A P K 1, C C N E 1$ and KRAS, are amplified in at least $10 \%$ of the cases (Supplementary Table 5.3).

GISTIC also identified 50 focal deletions (Fig. 1c). The known tumour suppressor genes PTEN, RB1 and NF1 were in regions of homozygous deletions in at least $2 \%$ of the tumours. Notably, $R B 1$ and NF1 also were among the significantly mutated genes. One deletion contained only three genes, including the essential cell cycle control gene $C R E B B P$, which has five non-synonymous and two reading frame shift mutations.

\section{mRNA and miRNA expression and DNA methylation analysis}

We combined expression measurements for 11,864 genes from three different platforms (Agilent, Affymetrix HuEx and Affymetrix U133A) for subtype identification and outcome prediction. Individual platform measurements suffered from limited, but statistically significant, batch effects, whereas the combined data set did not (Supplementary Methods, section 11, and Supplementary Fig. 11.1). Analysis of the combined data set identified $\sim 1,500$ intrinsically variable genes ${ }^{24}$ (Supplementary Methods, section 6) that were used for non-negative matrix factorization consensus clustering. This analysis yielded four clusters (Fig. 2a and Supplementary Methods, section 6). The same analytic approach applied to a publicly available data set from ref. 25 also yielded four clusters. Comparison of these two sets of four clusters showed a clear correlation (Supplementary Methods, section 6, and Supplementary Fig. 6.3). We therefore conclude that at least four robust expression subtypes exist in HGS-OvCa. 

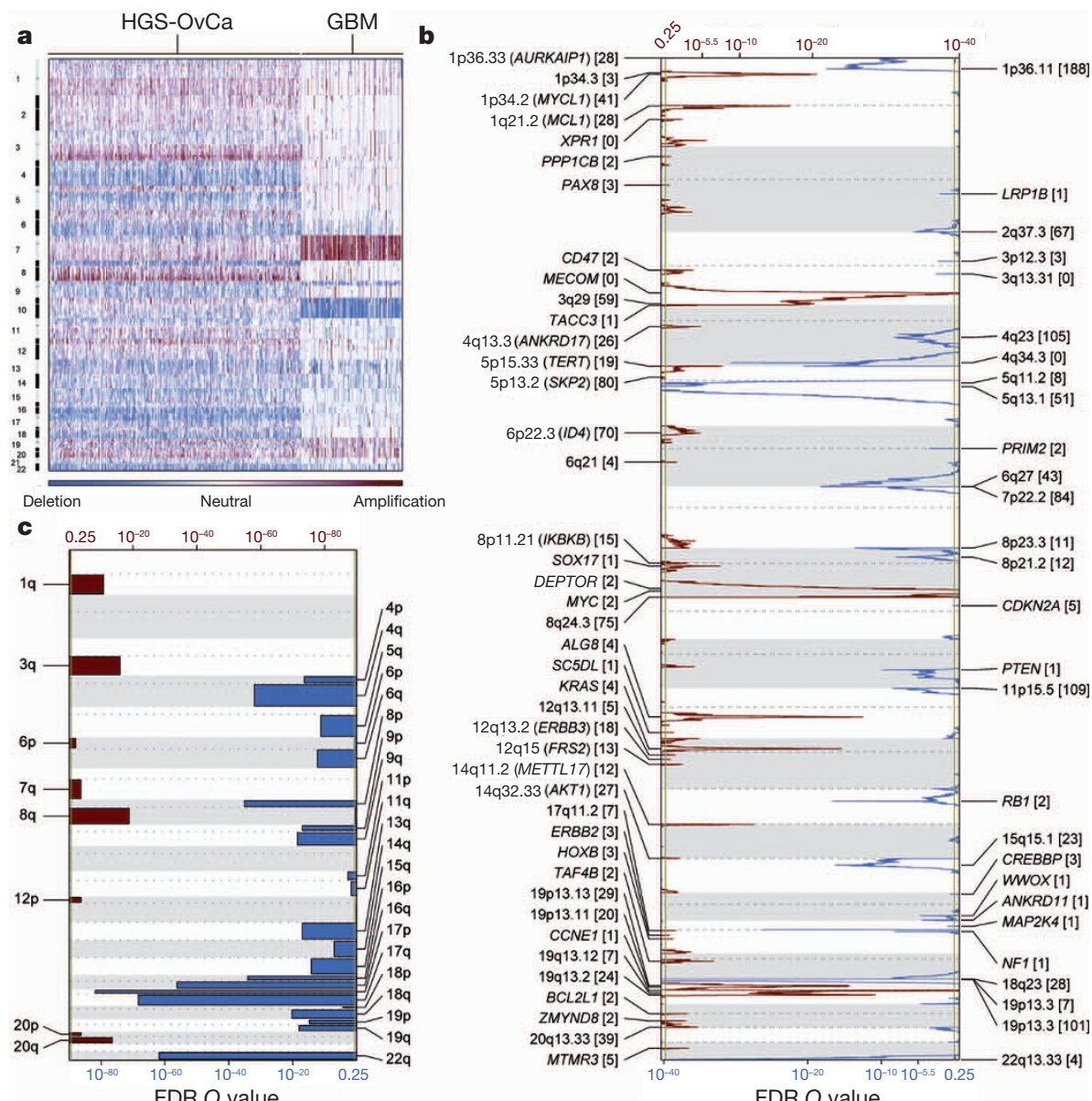

significant amplified and deleted regions, well-localized regions with eight or

Figure $1 \mid$ Genome copy number abnormalities. a, Copy number profiles of 489 HGS-OvCa, compared with profiles of 197 glioblastoma multiforme (GBM) tumours ${ }^{47}$. Copy number increases (red) and decreases (blue) are plotted as a function of distance along the normal genome (vertical axis, divided into chromosomes). b. Significant, focally amplified (red) and deleted (blue) regions are plotted along the genome. Annotations include the 20 most

We termed the four HGS-OvCa subtypes 'immunoreactive', 'differentiated', 'proliferative' and 'mesenchymal' on the basis of gene content in the clusters (Supplementary Methods, section 6) and previous observations $\mathrm{s}^{25}$. T-cell chemokine ligands CXCL11 and CXCL10 and the receptor CXCR3 characterized the immunoreactive subtype. High expression of transcription factors such as HMGA2 and SOX11, low expression of ovarian tumour markers (MUC1 and MUC16) and high expression of proliferation markers such as MCM2 and PCNA defined the proliferative subtype. The differentiated subtype was associated with high expression of MUC16 and MUC1 and with expression of the secretory fallopian tube maker SLPI, suggesting a more mature stage of development. High expression of HOX genes and markers suggestive of increased stromal components such as for myofibroblasts (FAP) and microvascular pericytes (ANGPTL2 and ANGPTL1) characterized the mesenchymal subtype.

Increased DNA methylation and reduced tumour expression implicated 168 genes as epigenetically silenced in HGS-OvCa samples compared with fallopian tube controls ${ }^{26}$. DNA methylation was correlated with reduced gene expression across all samples (Supplementary Methods, section 7). AMT, CCL21 and SPARCL1 were noteworthy because they showed promoter hypermethylation in the vast majority of the tumours. Unexpectedly, $R A B 25$, previously reported to be amplified and overexpressed in ovarian $\operatorname{cancer}^{27}$, also seemed to be epigenetically silenced in a subset of tumours. The $B R C A 1$ promoter was hypermethylated and silenced in 56 of 489 (11.5\%) tumours, as previously reported ${ }^{28}$ (Supplementary Fig. 7.1). fewer genes, and regions with known cancer genes or genes identified by genome-wide loss-of-function screens. The number of genes included in each region is given in brackets. FDR, false-discovery rate. c, Significantly amplified (red) and deleted (blue) chromosome arms.

Consensus clustering of variable DNA methylation across tumours identified four subtypes (Supplementary Methods, section 7, and Supplementary Fig. 7.2) that were significantly associated with differences in age, BRCA inactivation events and survival (Supplementary Methods, section 7). However, the clusters demonstrated only modest stability.

Survival duration did not differ significantly for transcriptional subtypes in the TCGA data set. The proliferative group showed a decrease in the rate of $M Y C$ amplification and $R B 1$ deletion, whereas the immunoreactive subtype showed an increased frequency of 3q26.2 $(M E C O M)$ amplification (Supplementary Table 6.2 and Supplementary Fig. 6.4). A moderate, but significant, overlap between the DNA methylation clusters and gene expression subtypes was noted $\left(P<2.2 \times 10^{-16}\right.$, chi-squared test, adjusted Rand index of 0.07 ; Supplementary Methods, section 7, and Supplementary Table 7.6).

A 193-gene transcriptional signature predictive of overall survival was defined using the integrated expression data set from 215 samples. After univariate Cox regression analysis, we found that 108 genes were correlated with poor survival and that 85 were correlated with good survival ( $P$-value cut-off of 0.01; Supplementary Methods, section 6, and Supplementary Table 6.4). We validated the predictive power of this gene expression signature on an independent set of 255 TCGA samples (Fig. 2b) as well as on three independent expression data $\operatorname{sets}^{25,29,30}$. Each of the validation samples was assigned a prognostic gene score, reflecting the similarity between its expression profile and the prognostic gene signature ${ }^{31}$ (Supplementary Methods, section 6). 

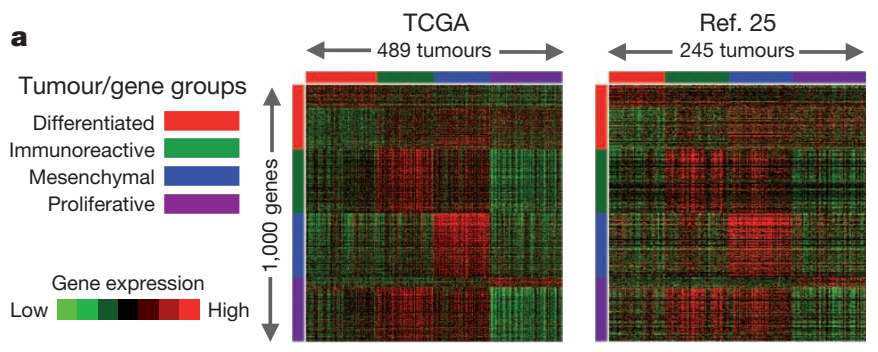

b

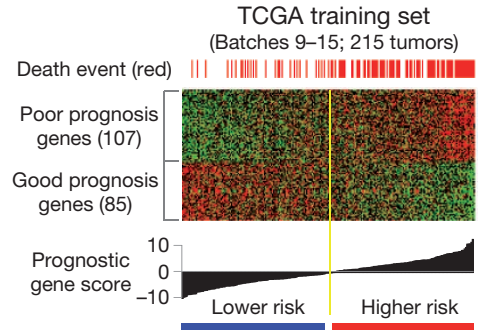

TCGA training set

TCGA test set (Batches 17-24; 255 tumors)

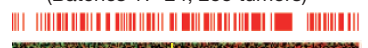
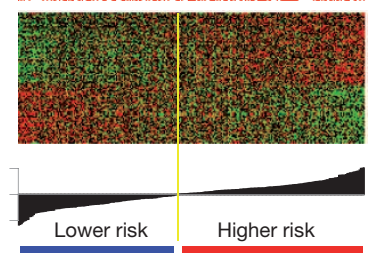

c
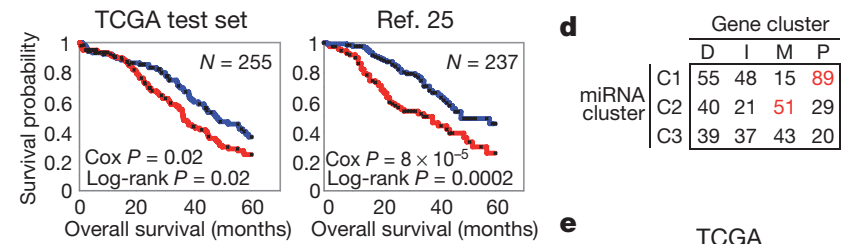

e
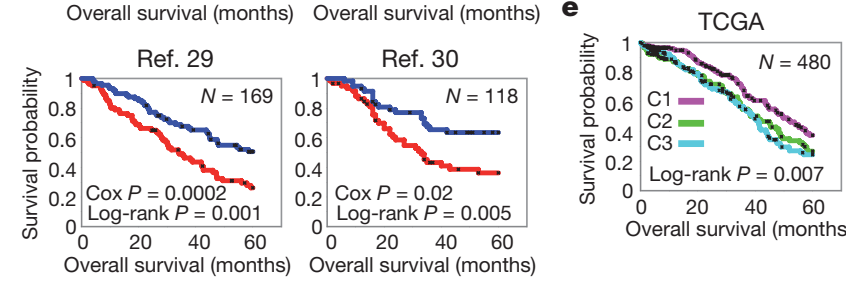

Figure $2 \mid$ Gene and miRNA expression patterns of molecular subtype and outcome prediction in HGS-OvCa. a, Tumours from TCGA and ref. 25 separated into four clusters on the basis of gene expression. $\mathbf{b}$, Using a training data set, a prognostic gene signature was defined and applied to a test data set. c, Kaplan-Meier analysis of four independent expression profile data sets, comparing survival for predicted higher-risk patients versus lower-risk patients. Univariate Cox $P$ value for risk index included. d, Tumours separated into three clusters on the basis of miRNA expression, overlapping with genebased clusters as indicated. D, differentiated; I, immunoreactive; M, mesenchymal; P, proliferative (red font indicates high degree of overlap). e, Differences in patient survival among the three miRNA-based clusters.

Kaplan-Meier survival analysis of this signature showed statistically significant association with survival in all validation data sets (Fig. $2 \mathrm{c}$ and Supplementary Methods, section 6).

Non-negative matrix factorization consensus clustering of miRNA expression data identified three subtypes (Supplementary Fig. 6.5). Notably, miRNA subtype 1 overlapped the mRNA proliferative subtype and miRNA subtype 2 overlapped the mRNA mesenchymal subtype (Fig. 2d). Survival duration differed significantly between miRNA subtypes: patients with miRNA subtype-1 tumours survived significantly longer (Fig. 2e).

\section{Pathways influencing disease}

Several analyses integrated data from the 316 fully analysed cases to identify biology that contributes to HGS-OvCa. Analysis of the frequency with which known cancer-associated pathways harboured one or more mutations, copy number changes or changes in gene expression showed that the RB1 and PI3K/RAS pathways were deregulated in $67 \%$ and $45 \%$ of cases, respectively (Fig. $3 \mathrm{a}$ and Supplementary Methods, section 8). A search for altered subnetworks in a large protein-protein interaction network ${ }^{32}$ using HOTNET $^{33}$ identified several known pathways (Supplementary Methods, section 9) including the NOTCH signalling pathway, which was altered in $22 \%$ of HGS-OvCa samples ${ }^{34}$ (Fig. 3b).

Published studies have shown that cells with mutated or methylated $B R C A 1$ or mutated BRCA2 have defective homologous recombination and are highly responsive to PARP inhibitors ${ }^{35-38}$. Fig. $3 \mathrm{c}$ shows that $20 \%$ of our studied HGS-OvCa samples had germline or somatic mutations in BRCA $1 / 2$, that $11 \%$ lost BRCA1 expression through DNA hypermethylation and that epigenetic silencing of $B R C A 1$ was mutually exclusive of $B R C A 1 / 2$ mutations $\left(P=4.4 \times 10^{-4}\right.$, Fisher's exact test $)$. Univariate survival analysis of $B R C A 1 / 2$ status (Fig. 3c) showed better overall survival for $B R C A 1 / 2$ mutated cases than $B R C A 1 / 2$ wild-type cases. Notably, epigenetically silenced $B R C A 1$ cases had survival similar to $B R C A 1 / 2$ wild-type HGS-OvCa tumours (respective median overall survivals of 41.5 and 41.9 months, $P=0.69$, log-rank test; Supplementary Methods, section 8 , and Supplementary Fig. 8.13b). This suggests that BRCA1 is inactivated by mutually exclusive genomic and epigenomic mechanisms and that patient survival depends on the mechanism of inactivation. Genomic alterations in other homologous recombination genes that might render cells sensitive to PARP inhibitors ${ }^{39}$ discovered in this study (Supplementary Methods, section 8, and Supplementary Fig. 8.12) include amplification or mutation of EMSY (also known as C11orf30) (8\%), focal deletion or mutation of PTEN (7\%), hypermethylation of RAD51C (3\%), mutation of ATM or ATR (2\%), and mutation of Fanconi anaemia genes (5\%). Overall, homologous recombination defects may be present in approximately half of all HGS-OvCa cases, providing a rationale for clinical trials of PARP inhibitors targeting tumours with these homologous-recombination-related aberrations.

Comparison between the complete set of BRCA inactivation events and all recurrently altered copy number peaks revealed an unexpectedly low frequency of CCNE1 amplification in cases with BRCA inactivation (8\% of BRCA altered cases had CCNE1 amplification whereas $26 \%$ of BRCA wild-type cases did; $Q=0.0048$, adjusted for false-discovery rate). As previously reported ${ }^{40}$, overall survival tended to be lower for patients with CCNE1 amplification than for patients in all other cases $(P=0.072$, log-rank test; Supplementary Methods, section 8, and Supplementary Fig. 8.14a). However, no survival disadvantage for CCNE1-amplified cases $(P=0.24, \log$-rank test; Supplementary Methods, section 8, and Supplementary Fig. 8.14b) was apparent when looking only at BRCA wild-type cases, suggesting that the previously reported CCNE1 survival difference can be explained by the higher survival of BRCA-mutated cases.

Finally, we used a probabilistic graphical model (PARADIGM $\left.{ }^{41}\right)$ to search for altered pathways in the US National Cancer Institute Pathway Interaction Database ${ }^{42}$, and found that the FOXM1 transcription factor network (Fig. 3d) is significantly altered in $87 \%$ of cases (Supplementary Methods, section 10, and Supplementary Figs 10.1-10.3). FOXM1 and its proliferation-related target genes, AurB (AURKB), CCNB1, BIRC5, CDC25 and PLK1, were consistently overexpressed but not altered by DNA copy number changes, indicative of transcriptional regulation. TP53 represses FOXM1 after DNA damage ${ }^{43}$, suggesting that the high rate of TP53 mutation in HGS-OvCa contributes to FOXM1 overexpression. In other data sets, the FOXM1 pathway is significantly activated in tumours relative to adjacent epithelial tissue $^{44-46}$ (Supplementary Methods, section 10, and Supplementary Fig. 10.4) and is associated with HGS-OvCa ${ }^{22}$ (Supplementary Methods, section 10, and Supplementary Fig. 10.5).

\section{Discussion}

This TCGA study provides a large-scale integrative view of the aberrations in HGS-OvCa. Overall, the mutational spectrum was surprisingly simple. Mutations in TP53 predominated, occurring in at least $96 \%$ of HGS-OvCa samples; and BRCA1 and BRCA2 were mutated in $22 \%$ of tumours, owing to a combination of germline and somatic mutations. Seven other significantly mutated genes were identified, but only in $2-6 \%$ of HGS-OvCa samples. By contrast, HGS-OvCa demonstrates a remarkable degree of genomic disarray. The frequency of SCNAs 
a $\mathrm{RB}$ and $\mathrm{PI} 3 \mathrm{~K} / \mathrm{RAS}$ signalling

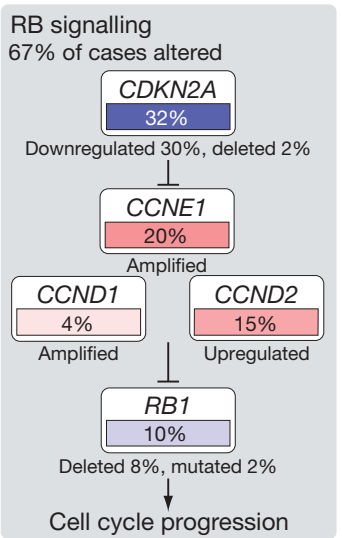

PI3K/RAS signalling

$45 \%$ of cases altered

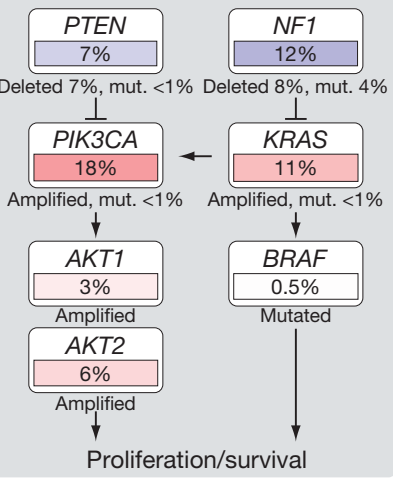

b $\mathrm{NOTCH}$ signalling

$22 \%$ of cases altered
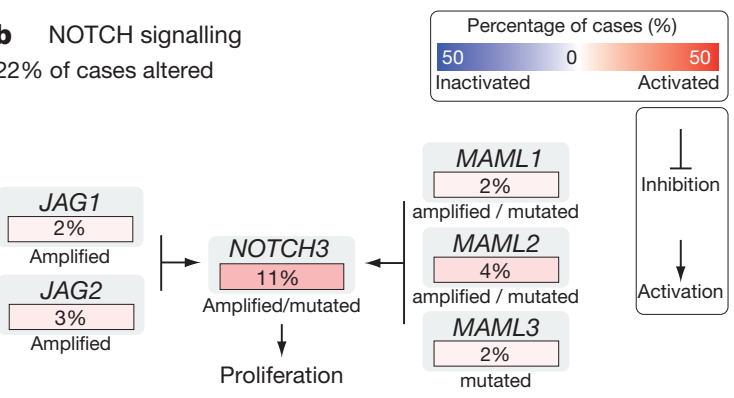

Figure $3 \mid$ Altered pathways in HGS-OvCa. a, b, RB and PI3K/RAS pathways, identified by curated analysis (a), and the NOTCH pathway, identified by HOTNET analysis (b), are commonly altered. Alterations are defined by somatic mutations, DNA copy number changes or, in some cases, by significant up- or downregulation relative to expression in diploid tumours. Alteration frequencies are expressed as a percentage of all cases; activated genes are red and inactivated genes are blue. c, Genes in the homologous recombination (HR) pathway are altered in up to $51 \%$ of cases. Survival analysis of BRCA1/2 status shows a divergent outcome for $B R C A 1 / 2$ mutated cases (with higher

stands in striking contrast to previous TCGA findings in glioblastoma ${ }^{47}$, where there were more recurrently mutated genes with far fewer chromosome arm-level or focal SCNAs (Fig. 1a). A high prevalence of mutations and promoter methylation in putative DNA repair genes, including homologous recombination components, may explain the high prevalence of SCNAs. The mutation spectrum marks HGS-OvCa as completely distinct from other ovarian cancer histological subtypes. For example, clear-cell ovarian cancer tumours have few TP53 mutations but have recurrent ARID1A and PIK3CA mutations ${ }^{48-50}$; endometrioid ovarian cancer tumours have frequent CTNNB1, ARID1A and PIK3CA mutations and a lower rate of TP53 (refs 49, 50); and mucinous ovarian cancer tumours have prevalent KRAS mutations ${ }^{51}$. These differences between ovarian cancer subtypes probably reflect a combination of aetiological and lineage effects, and represent an opportunity to improve ovarian cancer outcomes through subtype-stratified care.

Identification of new therapeutic approaches is a central goal of the TCGA. The $\sim 50 \%$ of HGS-OvCa tumours with homologous recombination defects may benefit from PARP inhibitors. Beyond this, the commonly deregulated pathways, RB, RAS/PI3K, FOXM1 and $\mathrm{NOTCH}$, provide opportunities for therapeutic treatment. Finally, inhibitors already exist for 22 genes in regions of recurrent amplification (Supplementary Methods, section 5, and Supplementary Table 5.3), warranting assessment in HGS-OvCa cases where the target genes are amplified. Overall, these discoveries set the stage for approaches to the treatment of HGS-OvCa in which aberrant genes or networks are detected and targeted with therapies selected to be effective against these specific aberrations. c HR alterations

BRCA altered cases, $N=103(33 \%)$

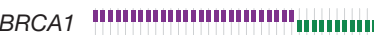

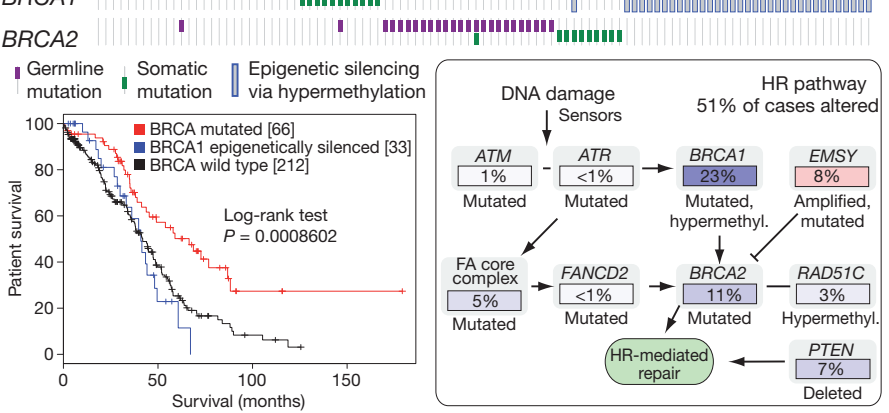

d FOXM1 signalling $\quad 84 \%$ of cases altered

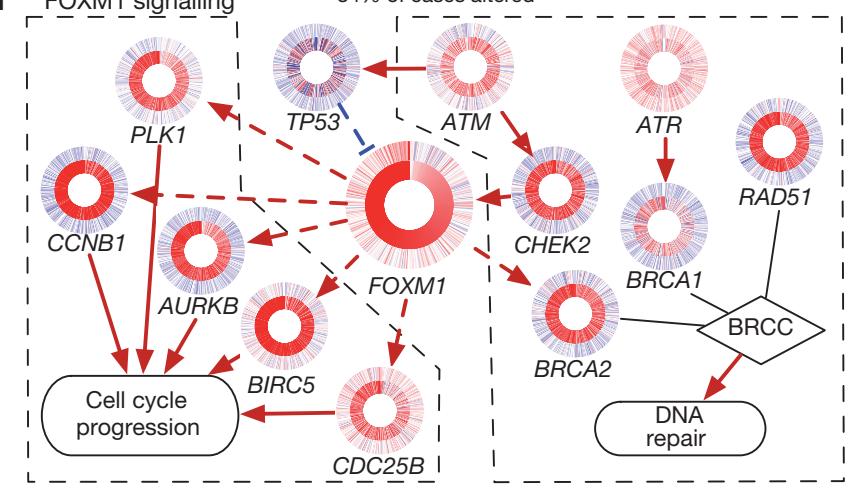

overall survival) than $B R C A 1 / 2$ wild type, and that $B R C A 1$ epigenetically silenced cases have poorer outcomes. FA, Fanconi anaemia. d, FOXM1 transcription factor network is activated in $84 \%$ of cases. Each gene is depicted as a multi-ring circle in which its copy number (outer ring) and gene expression (inner ring) are plotted such that each 'spoke' in the ring represents a single patient sample, with samples sorted in increasing order of FOXM1 expression. Excitatory interactions (red arrows) and inhibitory interactions (blue lines) were taken from the US National Cancer Institute Pathway Interaction Database. Dashed lines indicate transcriptional regulation.

\section{METHODS SUMMARY}

All specimens were obtained from patients with appropriate consent from the relevant institutional review board. DNA and RNA were collected from samples using the Allprep kit (Qiagen). We used commercial technology for capture and sequencing of exomes from whole-genome-amplified tumour DNA and normal DNA. DNA sequences were aligned to NCBI Build 36 of the human genome; duplicate reads were excluded from mutation calling. Validation of mutations occurred on a separate whole-genome amplification of DNA from the same tumour. Significantly mutated genes were identified by comparing them with expectation models based on the exact measured rates of specific sequence lesions. $\mathrm{CHASM}^{20}$ and MutationAssessor (Supplementary Methods, section 4) were used to identify functional mutations. GISTIC analysis of the circular-binary-segmented Agilent $1 \mathrm{M}$ feature copy number data was used to identify recurrent peaks by comparison with the results from the other platforms, to determine likely platform-specific artefacts. Consensus clustering approaches were used to analyse mRNA, miRNA and methylation subtypes as well as predictors of outcome using previous approaches ${ }^{47}$. HOTNET ${ }^{33}$ was used to identify portions of the proteinprotein interaction network that have more events than are expected by chance. Networks that had a significant probability of being valid were evaluated for increased fraction of known annotations. PARADIGM ${ }^{41}$ was used to estimate integrated pathway activity, to identify portions of the network models differentially active in HGS-OvCa.

\section{Received 7 September 2010; accepted 27 April 2011.}

1. Jemal, A., Siegel, R., Xu, J. \& Ward, E. Cancer statistics, 2010. CA Cancer J. Clin. 60, 277-300 (2010).

2. Koonings, P. P., Campbell, K., Mishell, D. R. Jr \& Grimes, D. A. Relative frequency of primary ovarian neoplasms: a 10-year review. Obstet. Gynecol. 74, 921-926 (1989).

3. Seidman, J. D. et al. The histologic type and stage distribution of ovarian carcinomas of surface epithelial origin. Int. J. Gynecol. Pathol. 23, 41-44 (2004). 
4. Miller, D. S. et al. Phase II evaluation of pemetrexed in the treatment of recurrent or persistent platinum-resistant ovarian or primary peritoneal carcinoma: a study of the Gynecologic Oncology Group. J. Clin. Oncol. 27, 2686-2691 (2009).

5. Jemal, A. et al. Cancer statistics, 2009. CA Cancer J. Clin. 59, 225-249 (2009)

6. Pal, T. et al. BRCA1 and BRCA2 mutations account for a large proportion of ovarian carcinoma cases. Cancer 104, 2807-2816 (2005).

7. Risch, H. A. et al. Population BRCA1 and BRCA2 mutation frequencies and cance penetrances: a kin-cohort study in Ontario, Canada. J. Natl Cancer Inst. 98, 1694-1706 (2006).

8. Bast, R. C. Jr, Hennessy, B. \& Mills, G. B. The biology of ovarian cancer: new opportunities for translation. Nature Rev. Cancer 9, 415-428 (2009).

9. Gnirke, A. et al. Solution hybrid selection with ultra-long oligonucleotides for massively parallel targeted sequencing. Nature Biotechnol. 27, 182-189 (2009).

10. Hodges, E. et al. Hybrid selection of discrete genomic intervals on customdesigned microarrays for massively parallel sequencing. Nature Protocols 4 , 960-974 (2009).

11. Bookman, M. A. et al. Evaluation of new platinum-based treatment regimens in advanced-stage ovarian cancer: a phase III trial of the Gynecologic Cancer Intergroup. J. Clin. Oncol. 27, 1419-1425 (2009)

12. Muggia, F. M. et al. Phase III randomized study of cisplatin versus paclitaxel versus cisplatin and paclitaxel in patients with suboptimal stage III or IV ovarian cancer: a gynecologic oncology group study. J. Clin. Oncol. 18, 106-115 (2000).

13. Ahmed, A. A. et al. Driver mutations in TP53 are ubiquitous in high grade serous carcinoma of the ovary. J. Pathol. 221, 49-56 (2010).

14. Chen, H. H., Wang, Y. C. \& Fann, M. J. Identification and characterization of the CDK12/cyclin L1 complex involved in alternative splicing regulation. Mol. Cell. Biol. 26, 2736-2745 (2006)

15. Ding, L. et al. Somatic mutations affect key pathways in lung adenocarcinoma Nature 455, 1069-1075 (2008).

16. Aldred, M. A. \& Trembath, R. C. Activating and inactivating mutations in the human GNAS1 gene. Hum. Mutat. 16, 183-189 (2000)

17. Forbes, S. A. et al. in Current Protocols in Human Genetics (eds Haines, J. L. et al.) Ch. 10, Unit 10.11 (Wiley, 2008).

18. McKusick, V. A. Mendelian Inheritance in Man and its online version, OMIM. Am. J. Hum. Genet. 80, 588-604 (2007).

19. Carter, H. etal. Cancer-specific high-throughput annotation of somatic mutations: computational prediction of driver missense mutations. Cancer Res. 69 , 6660-6667 (2009)

20. Carter, H., Samayoa, J., Hruban, R. H. \& Karchin, R. Prioritization of driver mutations in pancreatic cancer using cancer-specific high-throughput annotation of somatic mutations (CHASM). Cancer Biol. Ther. 10, 582-587 (2010).

21. Beroukhim, R. et al. The landscape of somatic copy-number alteration across human cancers. Nature 463, 899-905 (2010).

22. Etemadmoghadam, D. et al. Integrated genome-wide DNA copy number and expression analysis identifies distinct mechanisms of primary chemoresistance in ovarian carcinomas. Clin. Cancer Res. 15, 1417-1427 (2009).

23. Beroukhim, R. et al. Assessing the significance of chromosomal aberrations in cancer: methodology and application to glioma. Proc. Natl Acad. Sci. USA 104, 20007-20012 (2007).

24. Verhaak, R. G. et al. Integrated genomic analysis identifies clinically relevant subtypes of glioblastoma characterized by abnormalities in PDGFRA, IDH1, EGFR, and NF1. Cancer Cell 17, 98-110 (2010).

25. Tothill, R. W. et al. Novel molecular subtypes of serous and endometrioid ovarian cancer linked to clinical outcome. Clin. Cancer Res. 14, 5198-5208 (2008).

26. Dubeau, L. The cell of origin of ovarian epithelial tumours. Lancet Oncol. 9, 1191-1197 (2008)

27. Cheng, K. W. et al. The RAB25 small GTPase determines aggressiveness of ovarian and breast cancers. Nature Med. 10, 1251-1256 (2004).

28. Esteller, M. et al. Promoter hypermethylation and BRCA1 inactivation in sporadic breast and ovarian tumors. J. Natl. Cancer Inst. 92, 564-569 (2000).

29. Bonome, T. et al. A gene signature predicting for survival in suboptimally debulked patients with ovarian cancer. Cancer Res. 68, 5478-5486 (2008)

30. Dressman, H. K. et al. An integrated genomic-based approach to individualized treatment of patients with advanced-stage ovarian cancer. J. Clin. Oncol. 25, 517-525 (2007).

31. Creighton, C. J. et al. Insulin-like growth factor-I activates gene transcription programs strongly associated with poor breast cancer prognosis. J. Clin. Oncol. 26 4078-4085 (2008)

32. Keshava Prasad, T. S. et al. Human Protein Reference Database-2009 update Nucleic Acids Res. 37, D767-D772 (2009)

33. Vandin, F., Upfal, E. \& Raphael, B. J. in Proc. 14th Internat. Conf. Res. Comput. Mol. Biol. (ed. Berger, B.) 506-521 (Springer, 2010).

34. Choi, J. H. et al. Jagged-1 and Notch3 juxtacrine loop regulates ovarian tumor growth and adhesion. Cancer Res. 68, 5716-5723 (2008).

35. Farmer, $\mathrm{H}$. et al. Targeting the DNA repair defect in BRCA mutant cells as a therapeutic strategy. Nature 434, 917-921 (2005).

36. Fong, P. C. et al. Inhibition of poly(ADP-ribose) polymerase in tumors from BRCA mutation carriers. N. Engl. J. Med. 361, 123-134 (2009).

37. Veeck, J. et al. BRCA1 CpG island hypermethylation predicts sensitivity to poly(adenosine diphosphate)-ribose polymerase inhibitors. J. Clin. Oncol. 28, e563-e564 (2010)

38. Yap, T. A, et al. Reply to J. Veeck et al. J. Clin. Oncol. 10, e565-e566 (2010)

39. Mendes-Pereira, A. M. et al. Synthetic lethal targeting of PTEN mutant cells with PARP inhibitors. EMBO Mol. Med. 1, 315-322 (2009).

40. Nakayama, N. et al. Gene amplification CCNE1 is related to poor survival and potential therapeutic target in ovarian cancer. Cancer 116, 2621-2634 (2010).
41. Vaske, C. J. et al. Inference of patient-specific pathway activities from multidimensional cancer genomics data using PARADIGM. Bioinformatics 26, i237-i245 (2010)

42. Schaefer, C. F. et al. PID: the Pathway Interaction Database. Nucleic Acids Res. 37, D674-D679 (2009).

43. Barsotti, A. M. \& Prives, C. Pro-proliferative FoxM1 is a target of p53-mediated repression. Oncogene 28, 4295-4305 (2009).

44. Tone, A. A. et al. Gene expression profiles of luteal phase fallopian tube epithelium from BRCA mutation carriers resemble high-grade serous carcinoma. Clin. Cancer Res. 14, 4067-4078 (2008)

45. Myatt, S. S. \& Lam, E. W. The emerging roles of forkhead box (Fox) proteins in cancer. Nature Rev. Cancer 7, 847-859 (2007)

46. Wang, I. C. et al. Deletion of Forkhead Box M1 transcription factor from respiratory epithelial cells inhibits pulmonary tumorigenesis. PLOS ONE 4, e6609 (2009).

47. The Cancer Genome Atlas Research Network. Comprehensive genomic characterization defines human glioblastoma genes and core pathways. Nature 455, 1061-1068 (2008).

48. Ho, E. S.-C. et al. p53 mutation is infrequent in clear cell carcinoma of the ovary. Gynecol. Oncol. 80, 189-193 (2001)

49. Wiegand, K. C. et al. ARID1A mutations in endometriosis-associated ovarian carcinomas. N. Engl. J. Med. 363, 1532-1543 (2010)

50. Kuo, K. T. et al. Frequent activating mutations of PIK3CA in ovarian clear cell carcinoma. Am. J. Pathol. 174, 1597-1601 (2009).

51. Cuatrecasas, M., Villanueva, A., Matias-Guiu, X. \& Prat, J. K-ras mutations in mucinous ovarian tumors: a clinicopathologic and molecular study of 95 cases. Cancer 79, 1581-1586 (1997).

Supplementary Information is linked to the online version of the paper at www.nature.com/nature.

Acknowledgements We thank J. Palchik, A. Mirick and Julia Zhang for administrative coordination of TCGA activities. This work was supported by the following grants from the USA National Institutes of Health: U54HG003067, U54HG003079, U54HG003273, U24CA126543, U24CA126544, U24CA126546, U24CA126551, U24CA126554, U24CA126561, U24CA126563, U24CA143840, U24CA143882, U24CA143731, U24CA143835, U24CA143845, U24CA143858, U24CA144025, U24CA143882, U24CA143866, U24CA143867, U24CA143848, U24CA143843 and R21CA135877.

Author Contributions The TCGA research network contributed collectively to this study. Biospecimens were provided by the tissue source sites and processed by the biospecimen core resource. Data generation and analyses were performed by the genome sequencing centres, cancer genome characterization centres and genome data analysis centres. All data were released through the data coordinating centre. Project activities were coordinated by the $\mathrm{NCl}$ and $\mathrm{NHGRI}$ project teams. We also acknowledge the following TCGA investigators who contributed substantively to the writing of this manuscript: P.T.S. (project leader); S.G., G.G., J.W.G. and E.R.M. (writing team); A.K. (databases); L.D., K.C., D.A.W., M.D.M., M.S.L., G.G., D.C.K., A.S., C. Sougnez, N.D., Q.Z., C.K., M.C.W., B.R., R.K., H.C., D.V., Jinghui Zhang., J.W.W., D.K. and G.B.M. (mutations); G.G., C.H.M., M.I., A.H.R., W.W., J. Zhu, H.B., J.L., S.L.C., M.S.L., N.S., A.O B.A.W., J.W.G. and M.M. (copy number); P.W.L., H.S. and D.J.W. (methylation); R.G.W.V., C.J.C., H.Z., D.A.L., V.W., K.A.H., J.N.W., E.P., A.B. and M.B. (expression); D.N.H., S.M., Y.D., P.H.G., D.A.L., N.S., R.S and A.K.S. (miRNA); C. Sander, E.C., N.S., G.C., B.S.T., D.A.L., Y.X D.H., J.M.S., C.J.V., S.C.B., B.J.R., F.V., P.W.L. and H.S. (pathways); S.B.B., L.C., R.A.G., R.K., M.L., E.S.L., M.M., R.M.M., C.M.P. and R.K.W. (general).

Author Information Sequence information reported here has been submitted to $\mathrm{dbGaP}$ under accession number PHS000178. Reprints and permissions information is available at www.nature.com/reprints. The authors declare no competing financial interests. Readers are welcome to comment on the online version of this article at www.nature.com/nature. Correspondence and requests for materials should be addressed to P.T.S. (spellmap@ohsu.edu).

The Cancer Genome Atlas Research Network (Participants are arranged by area of contribution and then by institution.)

Disease working group and tissue source sites D. Bell ${ }^{1}, A$. Berchuck ${ }^{2,3}, M$. Birrer ${ }^{4,5}, \mathrm{~J}$. Chien $^{6}$, D. W. Cramer ${ }^{7}$, F. Dao ${ }^{8}$, R. Dhir ${ }^{9}$, P. DiSaial ${ }^{10}$, H. Gabra ${ }^{11}$, P. Glenn ${ }^{12}$, A. K. Godwin $^{13}$, J. Gross ${ }^{14}$, L. Hartmann ${ }^{15}$, M. Huang ${ }^{16}$, D. G. Huntsman ${ }^{17}$, M. lacocca ${ }^{18}$, M Imielinski ${ }^{5}$. S. Kalloger ${ }^{17}$, B. Y. Karlan ${ }^{14,19}$, D. A. Levine ${ }^{8}$, G. B. Mills ${ }^{20}, 21^{1}$, C. Morrison ${ }^{22,23}$ D. Mutch ${ }^{24}$, N. Olvera $^{8}$, S. Orsulic ${ }^{14}$, K. Park ${ }^{25}$, N. Petrelli ${ }^{26}$, B. Rabeno ${ }^{18}$, J. S. Rader ${ }^{27}$, B I. Sikic ${ }^{28}$, K. Smith-McCune ${ }^{12}$, A. K. Sood ${ }^{29,30}$, D. Bowtell ${ }^{31}$, R. Penny ${ }^{32}$, J. R. Testa $3^{33}$

Genome sequencing centres: Baylor College of Medicine K. Chang ${ }^{34}, \mathrm{C}$. J. Creighton $^{34}$, H. H. Dinh ${ }^{34}$, J. A. Drummond ${ }^{34}$, G. Fowler ${ }^{34}$, P. Gunaratne ${ }^{34}$, A. C. Hawes $^{34}$, C. L. Kovar ${ }^{34}$, L. R. Lewis ${ }^{34}$, M. B. Morgan ${ }^{34}$, I. F. Newsham ${ }^{34}$, J. Santibanez ${ }^{34}$, J. G. Reid ${ }^{34}$, L. R. Trevino ${ }^{34}$, Y.-O. Wu ${ }^{34}$, M. Wang ${ }^{34}$, D. M. Muzny ${ }^{34}$, D. A. Wheeler ${ }^{34}$, R. A Gibbs $^{34}$; Broad Institute G. Getz ${ }^{35}$, M. S. Lawrence ${ }^{35}$, K. Cibulskis ${ }^{35,36}$, A. Y. Sivachenko ${ }^{35}$, C. Sougnez ${ }^{37}$, D. Voet ${ }^{35}$, J. Wilkinson ${ }^{38}$, T. Bloom ${ }^{39}$, K. Ardlie $^{35}$, T. Fennell $^{40}$, J. Baldwin ${ }^{38}$, R. Nichol ${ }^{38}$, S. Fisher ${ }^{38}$, S. Gabriel ${ }^{41}$, E. S. Lander ${ }^{35,42,43}$; Washington University in St Louis L. Ding ${ }^{44}$, R. S. Fulton ${ }^{44}$, D. C. Koboldt ${ }^{44}$, M. D. McLellan $^{44}$, T. Wylie ${ }^{44}$, J. Walker ${ }^{44}$, M. O'Laughlin ${ }^{44}$, D. J. Dooling ${ }^{44}$, L. Fulton ${ }^{44}$, R. Abbott ${ }^{44}$ N. D. Dees ${ }^{44}$ O. Zhang ${ }^{4}$ C. Kandoth ${ }^{44}$ M. Wendl ${ }^{44}$, W. Schierding ${ }^{44}$ ' D. Shen $^{44}$, C. C. Harris ${ }^{44}$, H. Schmidt ${ }^{44}$, J. Kalicki ${ }^{44}$, K. D. Delehaunty ${ }^{44}$, C. C. Fronick ${ }^{44}$, R. Demeter $^{44}$, L. Cook ${ }^{44}$, J. W. Wallis ${ }^{44}$, L. Lin ${ }^{44}$, V. J. Magrini ${ }^{44}$, J. S. Hodges ${ }^{44}$, J. M. 
Eldred $^{44}$, S. M. Smith ${ }^{44}$, C. S. Pohl ${ }^{44}$, F. Vandin ${ }^{45}$, E. Upfal ${ }^{45}$, B. J. Raphael ${ }^{45}$, G. M. Weinstock ${ }^{44}$, E. R. Mardis ${ }^{44,46}$, R. K. Wilson ${ }^{44,46}$

Cancer genome characterization centres: Broad Institute/Dana-Farber Cancer Institute M. Meyerson ${ }^{35,47,48}$, W. Winckler ${ }^{37}$, G. Getz $^{37}$, R. G. W. Verhaak ${ }^{35,47}$, S. L.

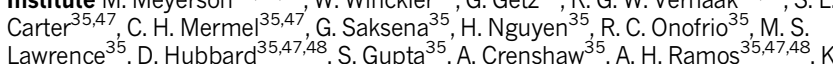
Lawrence 35 , D. Hubbard $35,47,48$, S. Gupta ${ }^{35}$, A. Crenshaw ${ }^{35}$, A. H. Ramos A $^{35,47,48}, \mathrm{~K}$. Kim $^{51}$, I. Perna ${ }^{49}$, Y. Xiao ${ }^{49}$, H. Zhang ${ }^{49}$, G. Ren ${ }^{49}$, N. Sathiamoorthy ${ }^{52}$, R. W. Park ${ }^{51}$, E. Lee ${ }^{51}$, P.J. Park ${ }^{51,53}$, R. Kucherlapati $i^{54}$; HudsonAlpha Institute/Stanford University D. University of Southern California/Johns Hopkins University P. W. Laird , $^{59}$, L. Cope ${ }^{60}$,J. G. Herman ${ }^{61}$, H. Shen ${ }^{59}$, D. J. Weisenberger ${ }^{59}$, H. Noushmehr ${ }^{59}$, F. Pan ${ }^{59}$, T. Triche Jr ${ }^{59}$, B. P. Berman ${ }^{59}$, D. J. Van Den Berg ${ }^{59}$, J. Buckley ${ }^{59}$, S. B. Baylin ${ }^{61}$; Lawrence Berkeley National Laboratory P. T. Spellman ${ }^{62}$, E. Purdom ${ }^{63}$, P. Neuvial ${ }^{63}$, H. Bengtsson ${ }^{63}$, L. R.

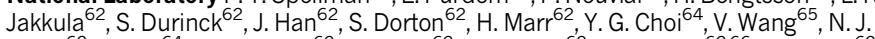
Wang ${ }^{62}$, J. Ngai ${ }^{64}$,J.G. Conboy ${ }^{62}$, B. Parvin ${ }^{62}$, H. S. Feiler ${ }^{62}$, T.P. Speed ${ }^{62,66}$, J.W. Gray ${ }^{62}$, Memorial Sloan-Kettering Cancer Center D. A. Levine ${ }^{8}$, N. D. Socci ${ }^{67}$, Y. Liang ${ }^{67}$, B. S Taylor $^{67}$, N. Schultz ${ }^{67}$, L. Borsu ${ }^{68}$, A. E. Lash ${ }^{67}$, C. Brennan ${ }^{69}$, A. Viale ${ }^{70}$, C. Sander ${ }^{67}$, M. Ladanyi ${ }^{68}$; University of North Carolina at Chapel Hill K. A. Hoadley ${ }^{71,72,73}$, S. Meng $^{73}$,

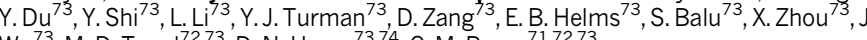

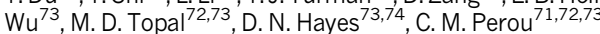

Genome data analysis centres: Broad Institute G. Getz ${ }^{35}$, D. Voet ${ }^{35}$, G. Saksena ${ }^{35}$ Junihua Zhang ${ }^{49}$, H. Zhang ${ }^{49}$, C. J. Wu ${ }^{49}$, S. Shukla 49 , K. Cibulskis ${ }^{35,36}$, M. S. Lawrence $^{35}$, A. Sivachenko ${ }^{35}$, R. Jing ${ }^{35}$, R. W. Park ${ }^{35,51}$, Y. Liu Li $^{49}$, P. J. Park ${ }^{51,53}$, M. Noble $^{35}$, L. Chin ${ }^{35,49,50}$; Johns Hopkins University H. Carter ${ }^{75}$, D. Kim ${ }^{75}$, J. Samayoa ${ }^{75}$ R. Karchin ${ }^{75}$; Lawrence Berkeley National Laboratory P. T. Spellman ${ }^{62}$, E. Purdom ${ }^{63}$ P. Neuvial ${ }^{63}$, H. Bengtsson ${ }^{63}$, S. Durinck ${ }^{62}$, J. Han ${ }^{62}$, J. E. Korkola ${ }^{62}$, L. M. Heiser ${ }^{62}$, R. J. $\mathrm{Cho}^{62}$, Z. Hu ${ }^{62}$, B. Parvin ${ }^{62}$, T. P. Speed ${ }^{62,66}$, J. W. Gray ${ }^{62}$; Memorial Sloan-Kettering Cancer Center N. Schultz ${ }^{67}$, E. Cerami ${ }^{67}$, B. S. Taylor ${ }^{67}$, A. Olshen ${ }^{76}$, B. Reva $a^{67}$ Y. Bernanke $^{78}$, L. Borsu ${ }^{68}$, D. A. Levine ${ }^{8}$, M. Ladanyi ${ }^{68}$, C. Sander ${ }^{67}$; University of California Santa Cruz/Buck Institute D. Haussler ${ }^{79,80}$, C. C. Benz $z^{81}$, J. M. Stuart ${ }^{79}$, S. C. Benz $^{79}$, J. Z. Sanborn ${ }^{79}$, C. J. Vaske ${ }^{79,82}$, J. Zhu ${ }^{79}$, C. Szeto ${ }^{79}$, G. K. Scott ${ }^{81}$, C. Yau ${ }^{81}$; University of North Carolina at Chapel Hill K. A. Hoadley ${ }^{71,72,73}$, Y. Du ${ }^{73}$, S. Balu ${ }^{73}$, D. N. Hayes $^{73,74}$, C. M. Perou ${ }^{71,72,73}$, M. D. Wilkerson ${ }^{73}$; The University of Texas MD Anderson Cancer Center N. Zhang ${ }^{83}$, R. Akbani ${ }^{83}$, K. A. Baggerly ${ }^{83}$, W. K. Yung ${ }^{84}$, G. B. Mills $^{20,21}$, J. N. Weinstein ${ }^{20,83}$

Biospecimen core resource R. Penny ${ }^{32}$, T. Shelton ${ }^{32}$, D. Grimm ${ }^{32}$, M. Hatfield ${ }^{32}$, S. Morris ${ }^{32}$, P. Yena ${ }^{32}$, P. Rhodes ${ }^{32}$, M. Sherman ${ }^{32}$, J. Paulauskis ${ }^{32}$, S. Millis ${ }^{32}$

Data coordination centre A. Kahn ${ }^{85}$, J.M. Mreene ${ }^{85}$, R. Sfeir ${ }^{85}$, M. A. Jensen ${ }^{85}$, J. Chen ${ }^{85}$, J. Whitmore ${ }^{85}$, S. Alonso ${ }^{85}$, J. Jordan ${ }^{85}$, A. Chu ${ }^{85}$, Jinghui Zhang ${ }^{86}$

Project teams: National Cancer Institute A. Barker ${ }^{87}$, C. Compton ${ }^{87}$, G. Eley ${ }^{87}$, M. Ferguson $^{88}$, P. Fielding ${ }^{87}$, D. S. Gerhard ${ }^{87}$, R. Myles ${ }^{87}$, C. Schaefer ${ }^{87}$, K. R. Mills Shaw ${ }^{87}$, J. Vaught ${ }^{87}$, J. G. Vockley ${ }^{87}$; National Human Genome Research Institute P. J. Good ${ }^{89}$ M. S. Guyer ${ }^{89}$, B. Ozenberger ${ }^{89}$, J. Peterson ${ }^{89}$ \& E. Thomson ${ }^{89}$

${ }^{1}$ Division of Anatomic Pathology, Mayo Clinic, Rochester, Minnesota 55905, USA. ${ }^{2}$ Division of Gynecologic Oncology/Department of Obstetrics and Gynecology, Duke University Medical Center, Durham, North Carolina 27708, USA. ${ }^{3}$ Duke Institute for Genome Sciences and Policy, Duke University Medical Center, Durham, North Carolina 27708, USA. ${ }^{4}$ Department of Medicine, Harvard Medical School, Boston, Massachusetts 02114, USA. ${ }^{5}$ Gynecologic Oncology, Massachusetts General Hospital, Boston, Massachusetts 02114 USA. ${ }^{6}$ Division of Experimental Pathology, Mayo Clinic, Rochester, Minnesota 55905, USA ${ }^{7}$ Department of Obstetrics and Gynecology Epidemiology Center, Brigham and Women's Hospital, Boston, Massachusetts 02115, USA. ${ }^{8}$ Department of Surgery, Memorial Sloan-Kettering Cancer Center, New York, New York 10065, USA. ${ }^{9}$ Department of Pathology, University of Pittsburgh, Pittsburgh, Pennsylvania 15213, USA. ${ }^{10}$ Gynecologic Oncology Group, University of California Irvine, Irvine, California 92697, USA. ${ }^{11}$ Ovarian Cancer Action Research Centre, Department of Surgery and Cancer, Imperial College London Hammersmith Campus, London W12 ONN, UK. ${ }^{12}$ Department of Obstetrics, Gynecology and Reproductive Services, University of California San Francisco, San Francisco, California 94143, USA. ${ }^{13}$ Women's Cancer Program, Department of Medical Oncology, Fox Chase Cancer Center, Philadelphia, Pennsylvania 19111, USA. ${ }^{14}$ Women's Cancer Research Institute at the Samuel Oschin Comprehensive Cancer Institute, Cedars-Sinai Medical Center, Geffen School of Medicine at UCLA, Los Angeles, California 90048, USA. ${ }^{15}$ Division of Medical Oncology, Mayo Clinic, Rochester, Minnesota 55905, USA. ${ }^{16}$ Department of Pathology, Fox Chase Cancer Center, Philadelphia, Pennsylvania 19111, USA. ${ }^{17}$ Center for Translational and Applied Genomics, British Columbia Cancer Agency, Vancouver, British Columbia V5Z 1G1, Canada. ${ }^{18}$ Department of Pathology, Christiana Care Health Services, Newark, Delaware 19718, USA. ${ }^{19}$ Department of Obstetrics and Gynecology, Cedars-Sinai Medical Center, Geffen School of Medicine at UCLA, Los Angeles, California 90048, USA. ${ }^{20}$ Department of Systems Biology, The University of Texas MD Anderson Cancer Center, Houston, Texas 77030, USA. ${ }^{21}$ Kleberg Center for Molecular Markers, The University of Texas MD Anderson Cancer Center, Houston, Texas 77030, USA. ${ }^{22}$ The Department of Pathology and Laboratory Medicine, Roswell Park Cancer Institute, Buffalo, New York 14263, USA. ${ }^{23}$ Division of Molecular Pathology, Roswell Park Cancer Institute, Buffalo, New York 14263, USA. ${ }^{24}$ Department of Obstetrics and Gynecology, Division of Gynecologic Oncology, Washington University School of Medicine in St Louis, St Louis, Missouri 63110, USA. ${ }^{25}$ Department of Pathology,
Memorial Sloan-Kettering Cancer Center, New York, New York 10065, USA. ${ }^{26}$ Department of Surgery, Helen F Graham Cancer Center at Christina Care, Newark, Delaware 19713, USA. ${ }^{27}$ Department of Obstetrics and Gynecology, Human and Molecular Genetics Center, Medical College of Wisconsin, Milwaukee, Wisconsin 53226, USA. ${ }^{28}$ Division of Oncology, Department of Medicine, Stanford University School of Medicine, Palo Alto, California 94304, USA. ${ }^{29}$ Department of Gynecologic Oncology, The University of Texas MD Anderson Cancer Center, Houston, Texas 77230, USA. ${ }^{30}$ Center for RNA Interference and Non-Coding RNA, The University of Texas MD Anderson Cancer Center, Houston, Texas 77230, USA. ${ }^{31}$ Research Division, Peter MacCallum Cancer Centre, Locked Bag 1 , A'Beckett St, Melbourne, Victoria 8006, Australia. ${ }^{32}$ International Genomics Consortium, Phoenix, Arizona 85004, USA. ${ }^{33}$ Cancer Biology Program, Fox Chase Cancer Center, Philadelphia, Pennsylvania 19111, USA. ${ }^{34}$ Human Genome Sequencing Center, Baylor College of Medicine, Houston, Texas 77030, USA. ${ }^{35}$ The Eli and Edythe L. Broad Institute of Massachusetts Institute of Technology and Harvard University, Cambridge, Massachusetts 02142, USA. ${ }^{36}$ Medical Sequencing Analysis and Informatics, The Eli and Edythe L. Broad Institute of Massachusetts Institute of Technology and Harvard University, Cambridge, Massachusetts 02142, USA. ${ }^{37}$ Cancer Genome \& Medical Resequencing Projects, The El and Edythe L. Broad Institute of Massachusetts Institute of Technology and Harvard University, Cambridge, Massachusetts 02142, USA. ${ }^{38}$ Sequencing Platform, The Eli and Edythe L. Broad Institute of Massachusetts Institute of Technology and Harvard University, Cambridge, Massachusetts 02142, USA. ${ }^{39}$ Sequencing Platform Informatics, The Eli and Edythe L. Broad Institute of Massachusetts Institute of Technology and Harvard University, Cambridge, Massachusetts 02142, USA. ${ }^{40}$ Directed Sequencing Informatics, The Eli and Edythe L. Broad Institute of Massachusetts Institute of Technology and Harvard University, Cambridge, Massachusetts 02142, USA. ${ }^{41}$ Genetic Analysis Platform, The Eli and Edythe L. Broad Institute of Massachusetts Institute of Technology and Harvard University, Cambridge, Massachusetts 02142, USA. ${ }^{42}$ Department of Biology, Massachusetts Institute of Technology, Cambridge, Massachusetts 02142, USA. ${ }^{43}$ Department of Systems Biology, Harvard University, Boston, Massachusetts 02115, USA. ${ }^{44}$ The Genome Center at Washington University, Department of Genetics, Washington University School of Medicine in St Louis, St Louis, Missouri 63108, USA. ${ }^{45}$ Department of Computer Science and Center for Computational Molecular Biology, Brown University, Providence, Rhode Island 02912 USA. ${ }^{46}$ Siteman Cancer Center, Washington University School of Medicine in St Louis, St Louis, Missouri 63108, USA. ${ }^{47}$ Department of Medical Oncology, Dana-Farber Cancer Institute, Boston, Massachusetts 02115, USA. ${ }^{48}$ Center for Cancer Genome Discovery, Dana-Farber Cancer Institute, Boston, Massachusetts 02115, USA ${ }^{49}$ Belfer Institute for Applied Cancer Science, Department of Medical Oncology, Dana-Farber Cancer Institute, Boston, Massachusetts 02115, USA. ${ }^{50}$ Department of Dermatology, Harvard Medical School, Boston, Massachusetts 02115, USA. ${ }^{51}$ The Center for Biomedical Informatics, Harvard Medical School, Boston, Massachusetts 02115, USA. ${ }^{52}$ Partners Center for Personalized Genetic Medicine, Cambridge, Massachusetts 02139, USA. ${ }^{53}$ Informatics Program, Children's Hospital, Boston, Massachusetts 02115, USA. ${ }^{54}$ Department of Genetics, Harvard Medical School, Boston, Massachusetts 02115, USA. ${ }^{55}$ HudsonAlpha Institute for Biotechnology, Huntsville, Alabama 35806, USA. ${ }^{56}$ Department of Genetics, Stanford University School of Medicine, Stanford, California 94305, USA. ${ }^{57}$ Department of Urology, Stanford University School of Medicine, Stanford, California 94305, USA. ${ }^{58}$ Department of Human Genetics, University of Michigan, Ann Arbor, Michigan 48109, USA. ${ }^{59}$ University of Southern California Epigenome Center, University of Southern California, Los Angeles, California 90089, USA. ${ }^{60}$ Biometry and Clinical Trials Division, The Sidney Kimmel Comprehensive Cancer Center at Johns Hopkins University, Baltimore, Maryland 21231, USA. ${ }^{61}$ Cancer Biology Division, The Sidney Kimmel Comprehensive Cancer Center at Johns Hopkins University, Baltimore, Maryland 21231, USA. ${ }^{2}$ Life Sciences Division, Lawrence Berkeley National Laboratory, Berkeley, California 94720, USA. ${ }^{63}$ Department of Statistics, University of California at Berkeley, Berkeley, California 95720, USA. ${ }^{64}$ Department of Molecular and Cellular Biology, University of California at Berkeley, Berkeley, California 95720, USA. ${ }^{65}$ Department of Biology and Biochemistry, University of Houston, Houston, Texas 77004, USA. ${ }^{66}$ Walter and Eliza Hall Institute, Parkville, Victoria 3052, Australia. ${ }^{67}$ Computational Biology Center, Memorial-Sloan Kettering Cancer Center, New York, New York 10065, USA. ${ }^{68}$ Department of Pathology, Human Oncology and Pathogenesis Program, Memorial-Sloan Kettering Cancer Center, New York, New York 10065, USA. ${ }^{69}$ Department of Neurosurgery, Memorial-Sloan Kettering Cancer Center, New York, New York 10065 , USA. ${ }^{70}$ Genomics Core Laboratory, Memorial-Sloan Kettering Cancer Center, New York, New York 10065, USA. ${ }^{71}$ Department of Genetics, University of North Carolina at Chapel Hill, Chapel Hill, North Carolina 27599, USA. ${ }^{72}$ Department of Pathology and Laboratory Medicine, Chapel Hill, North Carolina 27599, USA ${ }^{73}$ Lineberger Comprehensive Cancer Center, Chapel Hill, North Carolina 27599, USA. ${ }^{74}$ Department of Internal Medicine, Division of Medical Oncology, University of North Carolina at Chapel Hill, Chapel Hill, North Carolina 27599, USA. ${ }^{75}$ Institute for Computational Medicine, Department of Biomedical Engineering, Johns Hopkins University, Baltimore, Maryland 21231, USA ${ }^{76}$ Department of Epidemiology and Biostatistics, Memorial-Sloan Kettering Cancer Center, New York, New York 10065, USA. ${ }^{77}$ Department of Physiology and Biophysics, Weill Cornell Graduate School of Medical Sciences, New York, New York 10065, USA. ${ }^{78}$ Weill Medical College of Cornell University, 1300 York Avenue, New York, New York 10065, USA. ${ }^{79}$ Department of Biomolecular Engineering and Center for Biomolecular Science and Engineering, University of California Santa Cruz, Santa Cruz, California 95064, USA. ${ }^{80}$ Howard Hughes Medical Institute, University of California Santa Cruz, Santa Cruz, California 95064, USA. ${ }^{81}$ Buck Institute for Age Research, Novato, California 94945, USA. ${ }^{82}$ Lewis-Sigler Institute for Integrative Genomics, Princeton, New Jersey 08544, USA. ${ }^{83}$ Department of Bioinformatics and Computational Biology, The University of Texas MD Anderson Cancer Center, Houston, Texas 77030, USA. ${ }^{4}$ Department of Neuro-Oncology, The University of Texas MD Anderson Cancer Center, Houston, Texas 77030, USA. ${ }^{85}$ SRA International, Fairfax, Virginia 22033, USA. ${ }^{86}$ Department of Biotechnology, St Jude Children's Research Hospital, Memphis, Tennessee 38105, USA. ${ }^{87}$ National Cancer Institute, National Institutes of Health, Bethesda, Maryland 20892, USA. ${ }^{88}$ MLF Consulting, Arlington, Massachusetts 02474, USA. ${ }^{89}$ National Human Genome Research Institute, National Institutes of Health, Bethesda, Maryland 20892, USA. 
ERRATUM

doi:10.1038/nature11453

\section{Erratum: Integrated genomic analyses of ovarian carcinoma}

The Cancer Genome Atlas Research Network

Nature 474, 609-615 (2011); doi:10.1038/nature10166

In this Letter, J. G. Vockley was incorrectly listed as J. B. Vockley. Also, several authors were inadvertently omitted from the genome sequencing centre group: C. J. Creighton, from the Human Genome Sequencing Center at the Baylor College of Medicine; R. Nichol and S. Fisher, from the Sequencing Platform at the Broad Institute; and E. Upfal, from the Department of Computer Science at Brown University. In addition, J. Samayoa, from the Institute for Computational Medicine, was inadvertently missing from the genome data analysis centre group at the Johns Hopkins University. These have been corrected in the HTML and PDF versions of the manuscript. 